\title{
Preface
}

\section{Coordinate free numerics}

The four articles in this sequel are the results of a research initiative spearheaded by professors Magne Haveraaen and Hans Munthe-Kaas in Bergen, Norway. This initiative, sometimes recognized under the (viking) acronym of SAGA - Scientific computing and AlGebraic Abstraction, has subsequently spawned fruitful cooperation and further research, in part supported by the European Commission. Scientists in Swansea, Amsterdam, and Vilnius, among others, have contributed to further progress while exploring and developing the area.

This research brings previously somewhat distant disciplines from computer science and mathematics together focusing on important issues when designing modern software for scientific computing. The conflicts between higher level abstractions and compiler optimization techniques when applied to high performance numerical software is one example where this line of research may bring new progress.

The papers in this special issue, focusing on coordi- nate free numerical techniques, further explores:

- The changes higher level abstractions have on writing numerical software in Coordinate Free Programming of Computational Fluid Dynamics Problems,

- How programmer controlled, transparent parallel programming is facilitated via high level abstractions in Machine and Collection Abstractions for User-Implemented Data-Parallel Programming,

- The use of algebraic properties of software modules for optimization of scientific software in $A n$ Algebraic Programming Style for Numerical Software and its Optimization, and

- Tools for analysis and development of coordinate free software modules in Case Study on Algebraic Software Methodologies for Scientific Computing.

Petter E. Bjørstad Bergen, September 20, 2001 

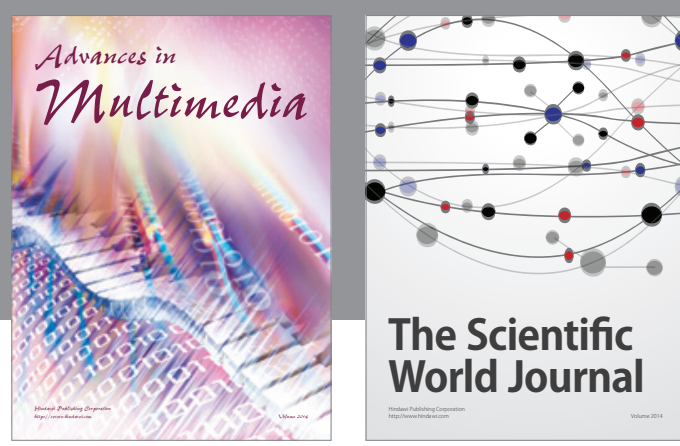

The Scientific World Journal
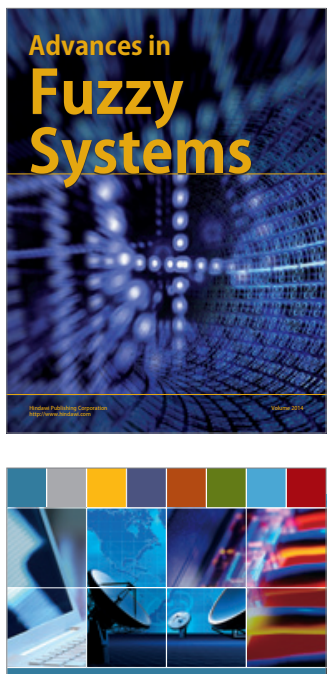

Computer Networks and Communications
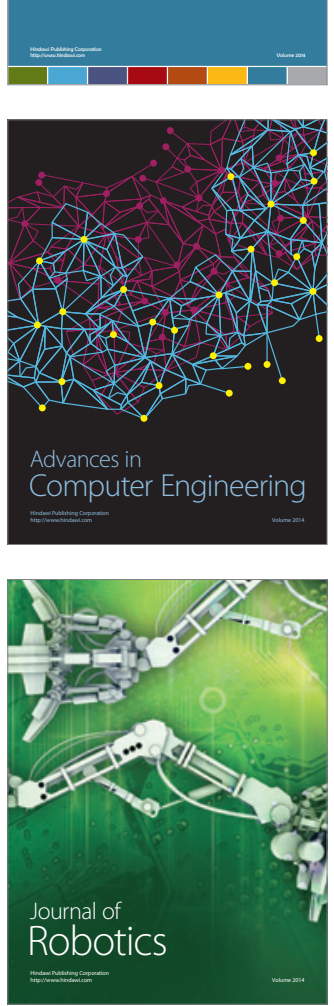
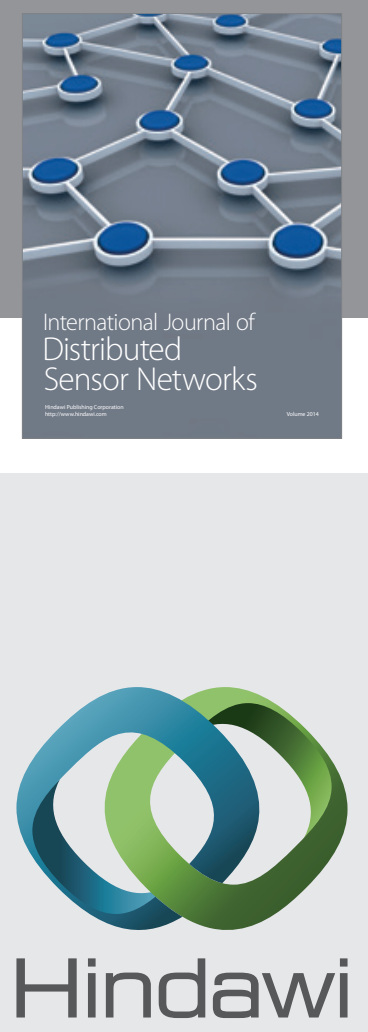

Submit your manuscripts at

http://www.hindawi.com
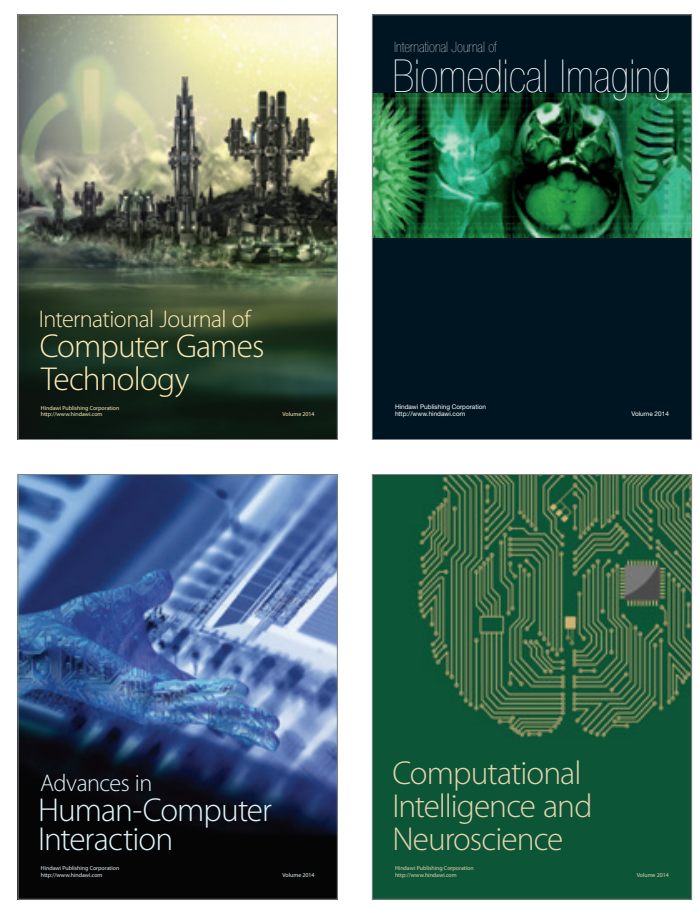
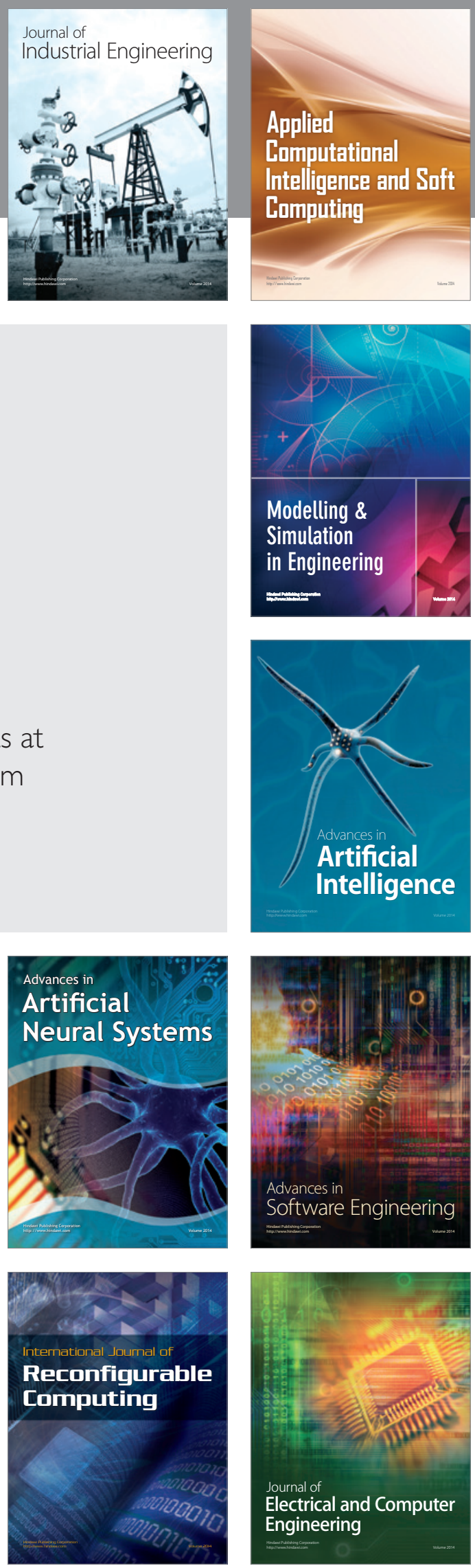